\title{
miR129-1 regulates protein phosphatase 1D protein expression under hypoxic conditions in non-small cell lung cancer cells harboring a TP53 mutation
}

\author{
HONG-LEI YIN, HONG-WEI XU and QING-YAN LIN \\ Department of Respiratory Medicine, Nangang Branch, Heilongjiang Provincial Hospital, \\ Harbin, Heilongjiang 150001, P.R. China
}

Received September 9, 2019; Accepted March 5, 2020

DOI: 10.3892/ol.2020.11783

\begin{abstract}
Protein phosphatase 1D (PPM1D), which functions as an oncogene, is a known target of the tumor suppressor p53 and is involved in p53-regulated genomic surveillance mechanisms. PPM1D dephosphorylates both p53 and its ubiquitin ligase mouse double minute 2 homolog, as well as the RNA-binding protein (RBM)38, which turns RBM38 from an inducer to inhibitor of TP53 translation. In addition, RBM38 induces PPM1D translation. Hence, the PPM1D-RBM38-p53 axis is important in maintaining genomic integrity and is often altered during tumorigenesis. TP53, which encodes $\mathrm{p} 53$, is deleted or mutated in $>50 \%$ of cancer types, including lung cancer. Mutant p53 has been revealed to complex with hypoxia-inducible factor $1 \alpha(\mathrm{HIF} 1 \alpha)$ and upregulate transcription of pro-metastatic genes. However, the mechanism underlying the action of the PPM1D-RBM38-p53 axis in the context of mutant p53 under normoxic and hypoxic conditions is yet to be elucidated. In the present study, using non-small cell lung cancer (NSCLC) cell lines harboring wild-type (A549 cells) or hot-spot mutant (NCI-H1770 and R249W $\Delta$-TP53-A549 cells) TP53, it was demonstrated that in cells harboring mutant p53, RBM38 was not the primary regulator of PPM1D translation under hypoxic conditions. Knockdown of RBM38 in TP53 mutant cells did not affect the PPM1D protein expression under hypoxic conditions. Instead, in NCI-H1770 cells maintained under normoxic conditions, $P P M 1 D$ was revealed as a target of micro RNA (miR)-129-1-3p, a known tumor suppressor in lung cancer. Hypoxia resulted in the downregulation of miR-129-1-3p expression, and thus, in the downregulation of PPMID messenger RNA (mRNA)
\end{abstract}

Correspondence to: Dr Qing-Yan Lin, Department of Respiratory Medicine, Nangang Branch, Heilongjiang Provincial Hospital, 405 Guogeli Street, Nangang, Harbin, Heilongjiang 150001, P.R. China E-mail: kathryn2190@163.com

Key words: p53, protein phosphatase 1D, RNA binding motif protein 38 , hypoxia-inducible factor $1-\alpha$, microRNA-129-1p, lung cancer translation. In NCI-H1770 cells grown under hypoxic conditions, the transient transfection of miR-129-1-3p mimic, and not control mimic, repressed the expression of a reporter containing wild-type, but not miR-129-1-3p binding mutant, of the PPM1D 3'-untranslated region (UTR). Analysis of NSCLC cell lines from the Broad Institute Cancer Cell Encyclopedia and patients with NSCLC from The Cancer Genome Atlas dataset revealed significant co-occurrence of $P P M 1 D / R B M 38$ and PPMID/HIF $1 A$ mutations. However, there was no significant difference in the overall survival of patients with NSCLC with or without genomic alterations in TP53, RBM38, $P P M 1 D$ and HIF1A. In summary, the current study demonstrated hypoxia-dependent miR-129-1-3p-mediated regulation of PPM1D protein expression in NSCLC cell line harboring mutant TP53.

\section{Introduction}

Wild-type p53-induced phosphatase 1D or PPM1D (also known as Wip1), a member of the PP2C serine/threonine phosphatase family, functions as an oncogene in multiple different types of cancer, including breast, esophageal, colon, thyroid, pancreatic, gastric, liver, nasopharyngeal, bladder, prostate and ovarian carcinoma (1-5). PPM1D exerts its oncogenic effect primarily via dephosphorylation and increased degradation of p53 protein $(6,7)$. Indeed, $P P M 1 D$ commonly exhibits copy number alterations and is upregulated in different types of cancer, including breast and ovarian carcinoma (2-5). Thus, the p53-PPM1D loop is an important mediator of the role of p53 in genomic surveillance mechanisms.

PPM1D protein upregulation in cancer cells is associated with a corresponding increase in mRNA expression. In fact, it has been revealed that the RNA-binding protein RBM38 or RNPC1 induces translation of $P P M 1 D$ by binding the 3'-untranslated region (UTR) of PPMID (8). Once translated, PPM1D protein dephosphorylates RBM38 at serine 195 residue (8). RBM38 is phosphorylated at the serine 195 residue by glycogen synthase kinase $3 \beta$ (GSK3 $\beta$ ). Dephosphorylated RBM38 forms a complex with the cap-binding protein eukaryotic elongation factor 4E (eIF4E) and prevents this from translating TP53 
mRNA $(9,10)$. Phosphorylation by GSK3 $\beta$ inhibits the interaction of RBM38 with eIF4E, resulting in translation of TP53 mRNA $(9,10)$. Thus, the PPM1D-RBM38-p53 axis is intricately regulated by feedback loops of kinases and phosphatases based on specific cellular context. Indeed, RBM38 is regulated by p53 and E2F1 $(11,12)$. RBM38 can also exert its pro-oncogenic roles by regulating mRNA stability and/or alternative splicing of cyclin-dependent kinase inhibitor $1 \mathrm{~A}$ (encoding p21), mouse double minute 2 homolog, ELAV-like RNA binding protein 1 (encoding human antigen R), erythrocyte membrane protein band 4.1 and fibroblast growth factor receptor $2(11,13,14)$.

The PPM1D-RBM38-p53 axis has been mostly studied in the context of wild-type p53. However, mutations in TP53 (both null and hot-spot point mutations) are known to exert gain-of-function that in turn regulate both resistance to chemotherapy and metastatic progression $(15,16)$, even though mutant TP53 is widely pervasive in all tumor types, including non-small cell lung cancer (NSCLC). In the case of T-cell lymphomagenesis, it has been demonstrated that RBM38 functions as a tumor suppressor, and genetic ablation of $R B M 38$ in a mice model resulted in enhanced mutant TP53 expression (17). A tumor is subjected to hypoxic conditions both during initial growth and during progression. However, it is not known whether the PPM1D-RBM38-p53 axis functions similarly under normoxic and hypoxic conditions. Given that RBM38 regulates cellular responses to oxidative stress by regulating translation of hypoxia-inducible factor $1 \alpha(H I F 1 A)(18)$, and HIF1 $\alpha$ protein forms a complex with mutant $\mathrm{p} 53$ protein and induces transcription of extracellular matrix components promoting migration and invasion (19), it may be possible that the PPM1D-RBM38-mutant p53 axis is regulated differently in normoxic and hypoxic conditions. Indeed, microRNAs (miRNAs), a class of small non-coding RNA 22 nucleotides long, have been shown to differentially regulate cellular response under hypoxic and normoxic conditions (20). miR-129-1-3p and miR-129-1-5p have been shown to function as tumor suppressors in colon, gastric, bladder, and esophageal cancer (21-24). However, to the best of our knowledge, it is unclear if miR-129-1 differentially regulates expression of PPM1D under normoxic or hypoxic conditions. Hence, the objective of the current study was to determine if PPM1D-RBM38-p53 axis is regulated similarly in NSCLC harboring wild-type and mutant p53 gene under the conditions of normoxia and hypoxia.

\section{Materials and methods}

Cell culture. A549 and NCI-H1770 cell lines were purchased from the American Type Culture Collection. Both cell lines were cultured in DMEM containing 10\% fetal bovine serum and $10 \mathrm{ml} / 1$ penicillin/streptomycin (all Thermo Fisher Scientific, Inc.). Cells were incubated in normoxic conditions $\left(21 \% \mathrm{O}_{2}, 74 \% \mathrm{~N}_{2}\right.$ and $\left.5 \% \mathrm{CO}_{2}\right)$ at $37^{\circ} \mathrm{C}$. For growth under hypoxia cells were incubated in a hypoxic chamber $\left(1 \% \mathrm{O}_{2}\right.$, $94 \% \mathrm{~N}_{2}$ and $5 \% \mathrm{CO}_{2}$; Jouan SA; Thermo Fisher Scientific, Inc.) at $37^{\circ} \mathrm{C}$ for $4 \mathrm{~h}$. During hypoxia induction, HEPES $(25 \mathrm{mM}$ final concentration; Thermo Fisher Scientific, Inc.) was added to prevent acidosis (25).
Cell lysate and western blot analysis. At the end of the experimental time point, the medium was removed and the cells were washed twice with ice-cold $1 \mathrm{X}$ phosphate-buffered saline (PBS). Cells were then lysed using RIPA buffer (20x volume of cell pellet; Thermo Fisher Scientific, Inc.), centrifuged at $15,000 \mathrm{x} \mathrm{g}$ for $15 \mathrm{~min}$ at $4^{\circ} \mathrm{C}$, and protein concentration in the extracted whole cell lysate was determined using a bicinchoninic acid assay (Thermo Fisher Scientific, Inc.). A total of $50 \mu \mathrm{g}$ of lysates per sample were resolved by $10 \%$ SDS-PAGE and blotted onto PVDF membranes (Thermo Fisher Scientific, Inc.) and processed for western blot analysis using standard methodologies. Blots were blocked using 5\% fat-free milk in TBST buffer (0/1\% Tween-20) for $30 \mathrm{~min}$ at room temperature before being incubated with the following primary antibodies overnight at $4^{\circ} \mathrm{C}$ : p53 (cat. no. 9282; 1:1,000; Cell Signaling Technology,Inc.),HA-Tag (cat.no. 3724; 1:1,500; Cell Signaling Technology, Inc.), PPM1D (cat. no. HPA022277; 1:2,000; Sigma-Aldrich; Merck KGaA), RBM38 (cat. no. ab168455; 1:3,000; Abcam), HIF1 $\alpha$ (cat. no. 36169; 1:1,000; Cell Signaling Technology, Inc.), p-p53 (serine 15) (cat. no. 9284; 1:1,000; Cell Signaling Technology, Inc.), GAPDH (cat. no. 5174; 1:4,000; Cell Signaling Technology, Inc.). Blots were probed with anti-GAPDH antibody to ensure equivalent protein loading across samples. After washing thrice with TBST buffer, blots were incubated with HRP-conjugated mouse or rabbit secondary antibody (Thermo Fisher Scientific, Inc.) for $1 \mathrm{~h}$ at room temperature. Post-incubation blots were washed thrice with TBST buffer before being developed using Pierce ECL Plus substrate (Thermo Fisher Scientific, Inc.). Each experiment was repeated $\geq$ three times and densitometry analysis was conducted using ImageJ version 2 software (National Institutes of Health) to determine the relative changes in protein expression under different conditions. Representative blots and quantification results from all experiments are presented in the figures.

miRNA isolation and reverse transcription quantitative $(R T-q P C R)$. Medium was aspirated off and cells were rinsed and scrapped off in ice-cold PBS and centrifuged at $1,000 \mathrm{x} \mathrm{g}$ for $5 \mathrm{~min}$ at $4^{\circ} \mathrm{C}$. The cell pellet was then used to isolate miRNA using the mirVana miRNA isolation kit (Thermo Fisher Scientific, Inc.). cDNA synthesis was performed using a TaqMan Advanced miRNA cDNA Synthesis kit (Thermo Fisher Scientific, Inc.). Each cDNA sample was pre-amplified using the TaqMan PreAmp Master Mix kit (Thermo Fisher Scientific, Inc.) then used to template the qPCR using the TaqMan Fast Advanced Master Mix and individual TaqMan microRNA assay probes (Thermo Fisher Scientific, Inc.). Thermocycling conditions consisted of an initial denaturation of $20 \mathrm{sec}$ at $95^{\circ} \mathrm{C}$, followed by 40 cycles of $95^{\circ} \mathrm{C}$ for $3 \mathrm{sec}$ and $60^{\circ} \mathrm{C}$ for $30 \mathrm{sec}$. qPCR was performed using TaqMan probes (Thermo Fisher Scientific, Inc.) for miR129-1 (Assay ID: 002298; 5'-AAGCCCUUACCCCAAAAAGUAU-3') and RNU6B (Assay ID: 001093). RNU6B expression levels were utilized for normalization. Post-normalization relative expression of miR129-1 was calculated using the $2^{-\Delta \Delta \mathrm{Cq}}$ method (26). The data were represented as expression in hypoxic conditions relative to normoxia, or expression following transfection of miR129-1 mimic compared with control mimic in hypoxia [mean \pm standard error of the mean (SEM)]. The RT-qPCR 
for the indicated genes was conducted similarly using TaqMan probes; however, the data were normalized to $G A P D H$. RT-qPCR experiments were run in triplicate.

Plasmid construction. The 3'-UTR of PPM1D was amplified from cDNA using the following primer sequences: PPM1D forward, 5'-TGCATCTGGGAAATGAGGTT-3' and reverse, 5'-GCCTCCTTCCAGATGACACT-3' and cloned into the pRL-TK-CXCR4 vector (Addgene, Inc.) and called the pRL-TK-PPM1D wild-type 3'-UTR plasmid. The miR-129-1 binding site mutant 3'-UTR (nucleotides 292-299 deleted) of PPMID was generated using site-directed mutagenesis (QuickChange II kit; Agilent Technologies, Inc.) and the following primers: Forward, 5'-GAGTCTCTGATACACAGT AATTGTGACAATATGTTTAAAGAAATCAAAAGAATC TATTA-3' and reverse, 5'-TAATAGATTCTTTTGATTTCT TTAAACATATTGTCACAATTACTGTGTATCAGAGAC TC-3' and named the pRL-TK- $\triangle P P M 1 D$ 3'-UTR plasmid. The pGL3 plasmid expressing Firefly luciferase off a CMV promoter was purchased from Promega Corporation. The miR129-1 (hsa-miR-129-1-3p) mimic used was the MISSION ${ }^{\circledR}$ microRNA mimic (cat. no. HMI0159; Sigma-Aldrich; Merck $\mathrm{KGaA}$ ) and the control mimic used was an oligonucleotide sequence from Arabidopsis thaliana (5'-GGUUCGUACGUA CACUGUUCA-3') with no homology to human gene sequences (cat. no. HMC0002; Sigma-Aldrich; Merck KGaA). Lentiviral particles of control short hairpin (sh)RNA (cat. no. sc-108080) and $R B M 38$ (cat. no. sc-76368-V) were obtained from Santa Cruz Biotechnology, Inc. shRNA targeting the 3'-UTR of TP53 (V2LHS_93615) was obtained from Open Biosystems. The pCMV-Neo-Bam p53 R248W plasmid (cat. no. 16437) was obtained from Addgene, Inc. and was cloned into pEF-hemagglutinin (HA) vector.

Transfection and transduction. Cells were plated in respective antibiotic-free cell culture DMEM medium. Cells were co-transfected with Renilla luciferase reporter plasmids [pRL-TK-PPM1D wild-type 3'-UTR or pRL-TK- $\triangle P P M 1 D$ 3'-UTR (nucleotides 292-299 deleted)] and pGL3 plasmid expressing Firefly luciferase, and where indicated along with control or miR129-1 mimic using Polyplus jetPRIME transfection reagent (Polyplus-transfection, SA). For reporter plasmids, cells were seeded in 24-well plates $(50,000$ cells/well) and co-transfected with $0.5 \mu \mathrm{g}$ of pRL-TK plasmid(s) and pGL3 plasmid. Luciferase assays were performed $24 \mathrm{~h}$ after transfection. miRNA mimics were transfected at a final concentration of $10 \mathrm{nM}$. Cells were harvested $48 \mathrm{~h}$ after transfection with the miRNA mimics.

For transduction, NCI-H1770 cells were transduced with either lentiviral particles containing control shRNA or shRNA targeting RBM38, using polybrene. Cells were selected with puromycin $(2 \mu \mathrm{g} / \mathrm{ml})$ for 2 weeks and successful knockdown was verified via western blot analysis.

A549 cells were first transfected with linearized pEF-HA-TP53 (R248W) plasmid and selected with G418 $(100 \mu \mathrm{g} / \mathrm{ml}$ ) for 2 weeks (with addition of fresh media every third day) before being transfected with lentiviral particles containing control shRNA or shRNA targeting 3'-UTR of TP53 using polybrene. Cells were selected with puromycin $(2 \mu \mathrm{g} / \mathrm{ml}$ ) for 2 weeks (with addition of fresh media every alternate day) and successful knockdown of endogenous and overexpression of the mutant TP53 plasmids was verified by western blot analysis using p53 and HA antibodies.

Luciferase assay. After $24 \mathrm{~h}$ of transfection, NCI-H1770 cells were washed with ice-cold PBS and then incubated with $100 \mu \mathrm{l}$ of $1 \mathrm{X}$ passive lysis buffer (Promega Corporation). Plates were incubated on a rocker at $37^{\circ} \mathrm{C}$ for $30 \mathrm{~min}$. After incubation, $20 \mu \mathrm{l}$ of lysates were used to perform a luciferase assay using the Dual-Luciferase assay kit (Promega Corporation). Firefly luciferase was used as an internal control and used to normalize relative Renilla luciferase expression and expressed as the mean \pm SD of three independent experiments.

Data mining. The analysis of The Cancer Genome Atlas (TCGA) (cancer.gov/tcga) data was performed using cBioPortal for Cancer Genomics (http://cbioportal.org) $(27,28)$. A total of 2,628 patients and 2,855 samples from 7 studies were included (29-35). Analysis was carried out to determine genome amplification, somatic mutations, association with survival and mRNA expression. In situ prediction of miRNAs binding to PPMID mRNA was done using TargetScan Human Release 7.1 algorithm (36).

Statistical analysis. Statistical analysis was performed using GraphPad Prism 6.0 (GraphPad Software, Inc.). Data were expressed as the mean \pm standard deviation of three technical replicates. For RT-qPCR, the data were expressed as the mean \pm SEM of three technical replicates. A paired Student's t-test was used to test differences between groups. The log-rank test was used to test whether the difference between overall survival times between two groups was statistically significant. $\mathrm{P}<0.05$ was considered to indicate a statistically significant difference.

\section{Results}

PPM1D protein expression is regulated independent of RBM38 expression in NSCLC cell line harboring mutant TP53. The protein expression levels of p53, PPM1D and RBM38 were initially determined in the NSCLC cell line A549 that harbors wild-type TP53, and in the NCI-H1770 cell line that harbors the R248W hotspot TP53 mutation. Although RBM38 and PPM1D expression levels were higher in the NCI-H1770 cells, p53 expression was higher in A549 cells (Fig. 1A). The higher basal expression of p53 observed in NCI-H1770 cells in comparison with the A549 cells was opposite to what is reported by the American Type Culture Collection (atcc.org/en/Documents/Learning_ Center/ /media/5F7B1CCACF724E3398BE56BFBEE3EFE4. ashx). Given that the objective of the present study was to define the regulation of the PPM1D-RBM38-p53 (wild-type/mutant) axis in the cells, this discordance in basal p53 expression was not further investigated.

RBM38 promotes translation of mutant TP53 (9). Mutant p53 protein cooperates with HIF1 $\alpha$ to cause transcriptional upregulation of extracellular matrix components (18). RBM38 can also regulate translation of HIF1 $\alpha$ (15). Hence, the changes in protein expression of PPM1D, p53, and RBM38 following 

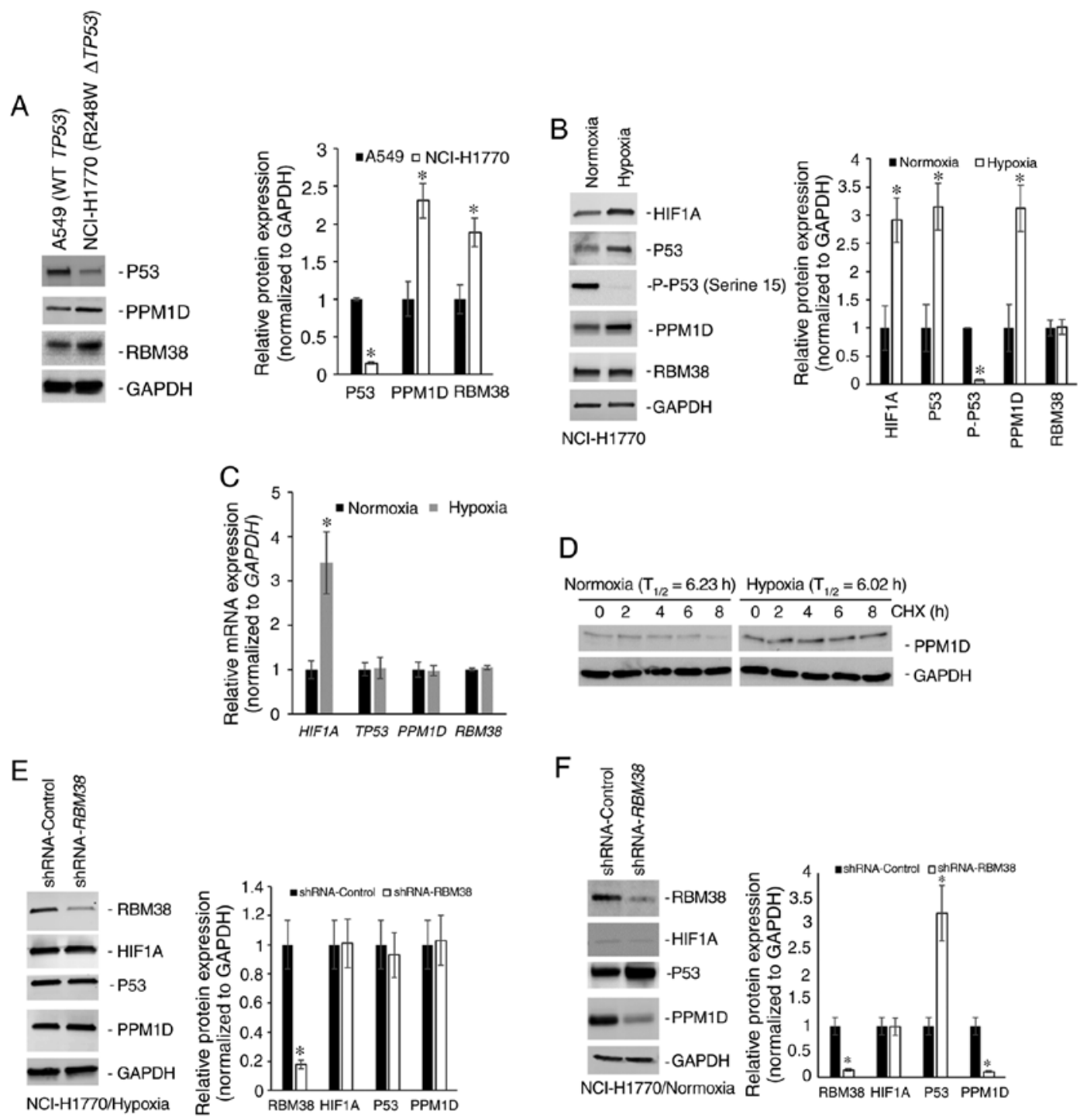

Figure 1. PPM1D protein expression is regulated independently of RBM38 expression in NCI-H1770 cells harboring R248W mutant TP53. (A) Western blot analysis of indicated proteins in A549 and NCI-H1770 cell lines maintained under normoxic conditions. The bar graph presents the relative protein expression levels determined by densitometry analysis $(\mathrm{n}=3)$ and expressed as the mean \pm standard deviation. (B) Western blot analysis of indicated proteins in NCI-H1770 cells maintained under normoxic conditions or subjected to hypoxia for $4 \mathrm{~h}$. The bar graph presents the relative protein expression levels determined by densitometry analysis $(n=3)$ and expressed as the mean \pm standard deviation. (C) Relative mRNA expression of indicated genes. Data were normalized to GAPDH and expressed as the mean \pm standard error of the mean relative to normoxia $(\mathrm{n}=3)$. (D) Relative stability of PPM1D protein in normoxic and hypoxic conditions as determined by CHX treatment. $\mathrm{T}_{1 / 2}$ was calculated from three replicates and normalized to GAPDH. Western blot analysis of indicated proteins in NCI-H1770 cells stably transduced with either control shRNA or shRNA targeting RBM38 and maintained under (E) hypoxia for 4 h, or (F) normoxia. Bar graphs in indicate the relative protein expression levels determined by densitometry analysis $(n=3)$ and expressed as the mean \pm standard deviation. Blots in each case (A, B, E and F) were probed with anti-GAPDH antibody to confirm equal loading. " $\mathrm{P}<0.05$. mRNA, messenger RNA; $\mathrm{T}_{1 / 2}$, half-life; PPM1D, p53-induced phosphatase 1D; shRNA, short hairpin RNA; RBM38, RNA binding motif protein 38; WT, wild type; CHX, cycloheximide; HIF1A, hypoxia-inducible factor $1 \mathrm{~A}$.

induction of hypoxia in the NCI-H1770 cells were investigated. Hypoxia was induced by incubating cells at $1 \% \mathrm{O}_{2}$ for $4 \mathrm{~h}$ and confirmed via the induction of HIFl $\alpha$ protein expression. RBM38 protein expression did not change following the induction of hypoxia in these cells; however, both p53 and PPM1D were upregulated following hypoxia (Fig. 1B). Phosphorylation levels of p53 at serine 15 residue decreased following the induction of hypoxia (Fig. 1B), which may have been either due to decreased phosphorylation or increased phosphatase activity, given the increase in PPM1D protein expression. The increase in protein expression of PPM1D and p53 was not due to transcriptional upregulation post-hypoxia, as the steady state mRNA expression level did not differ significantly between normoxic and hypoxic conditions (Fig. 1C). To determine whether the increased PPM1D protein expression in hypoxia was due to increased stability of the protein, cycloheximide chase experiment was performed, as cycloheximide inhibits translation. PPM1D half-life $\left(\mathrm{T}_{1 / 2}\right)$ was not significantly different between normoxic $(6.23 \mathrm{~h})$ and hypoxic conditions (6.02 h) (Fig. 1D). Cumulatively, these results indicate that PPM1D and $\mathrm{p} 53$ protein expression was post-transcriptionally upregulated during hypoxia in the NCI-H1770 cells.

Given that no change was observed in RBM38 protein expression between normoxic and hypoxic conditions, there are two possible explanations for the observed induction of p53 and PPM1D proteins (Fig. 1B). The first one is that the 
A
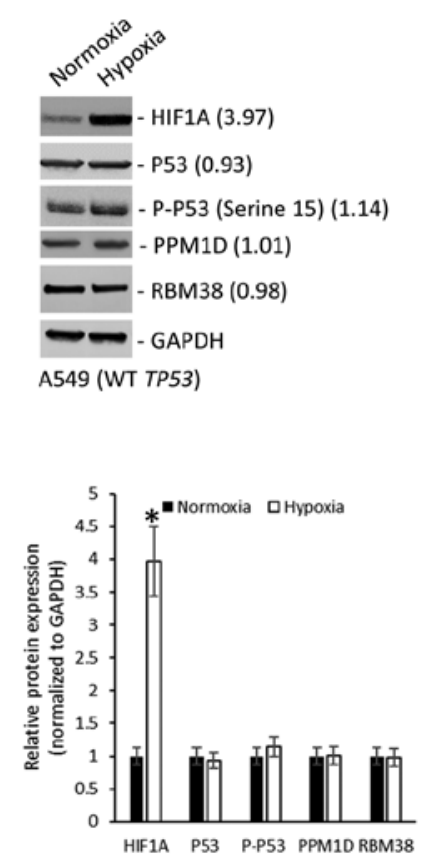

B

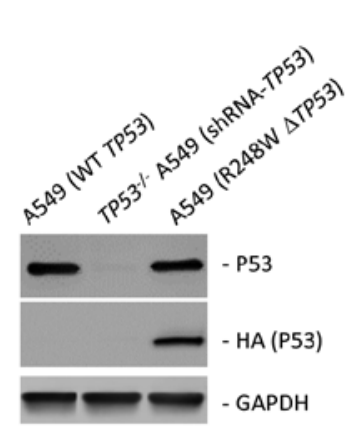

C

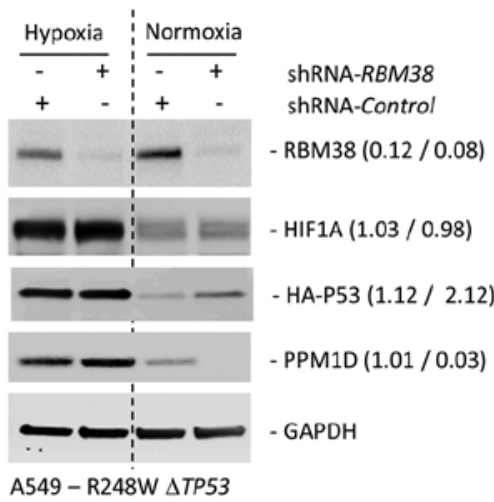

Figure 2. Differential regulation of PPM1D in A549 cells harboring wild-type or mutant TP53. (A) Western blot analysis of indicated proteins in parental A549 cells (harboring wild-type TP53) maintained under normoxic conditions or subjected to hypoxia for $4 \mathrm{~h}$. The bar graph presents the relative expression levels determined by densitometry analysis $(n=3)$ and data are expressed as the mean \pm standard deviation. (B) A549 cells were transfected with pEF-HA-tagged R248W TP53 and stable cells were then transduced with shRNA targeting 3'-untranslated region of TP53. Knockdown of endogenous TP53 and expression of the mutant plasmid was verified via western blot analysis. (C) Western blot analysis of indicated proteins in A549-R248W TP53 cells stably transduced with either control shRNA or shRNA targeting RBM38 and maintained under hypoxic conditions for $4 \mathrm{~h}$ or under normoxic conditions. The bar graph displays the relative expression levels determined by densitometry analysis $(n=3)$ and expressed as the mean \pm standard deviation. Blots in each panel were probed with anti-GAPDH antibody to confirm equal loading. "P<0.05. PPM1D, p53-induced phosphatase 1D; shRNA, short hairpin RNA; RBM38, RNA binding motif protein 38; WT, wild type; p-, phosphorylated; HIF1 $\alpha$, hypoxia-inducible factor $1 \alpha$; HA, hemagglutinin.

binding of RBM38 to PPM1D's 3'-UTR is increasing under hypoxic conditions, which results in the increased translation of PPMID mRNA, and the translated PPM1D is then dephosphorylating RBM38 protein, inducing the translation of mutant TP53 mRNA. Alternatively, there is a secondary mechanism of regulating PPM1D expression under hypoxic conditions. To test the first possible explanation, NCI-H1770 variants stably expressing either a control shRNA or shRNA targeting RBM38 were generated. Successful knockdown was verified by western blot analysis (Fig. 1E). Cells expressing the control or $R B M 38$ shRNA were subjected to hypoxia. Even though it has been shown before that RBM38 regulates translation of HIF1A mRNA (15), knockdown of RBM38 did not affect HIF1 $\alpha$ protein induction (Fig. 1E). This was not surprising given that HIF1 $\alpha$ is known to be regulated at multiple different levels. $R B M 38$ knockdown did not affect the induction in protein expression of PPM1D or mutant p53 (Fig. 1E). However, when the same experiment was repeated under normoxic conditions, knockdown of RBM38 resulted in a significant decrease in PPM1D protein expression, and an increase in p53 protein expression (Fig. 1F), corroborating findings from other studies performed in normoxic conditions $(9,10,17)$. This indicated that alternate post-transcriptional regulatory mechanism(s) were regulating PPM1D expression under hypoxic conditions.

Differential regulation of PPM1D in A549 cells harboring wild-type or mutant TP53. To determine whether such a potential alternate regulatory mechanism of PPM1D protein expression operated in NSCLC cells harboring wild-type TP53, the A549 cells were subjected to hypoxia. Hypoxia was induced by incubating cells at $1 \% \mathrm{O}_{2}$ for $4 \mathrm{~h}$ and confirmed via the induction of HIF1 $\alpha$ protein expression (Fig. 2A). The protein expression of RBM38, p53, p-p53 (serine 15) and PPM1D was not significantly different under normoxic and hypoxic conditions (Fig. 2A), indicating a differential response to hypoxia in NSCLC cells harboring wild-type or mutant TP53. Given that A549 cells are epithelial cells from a lung tumor mass, whereas the NCI-H1770 cells are neuroendocrine cells derived from lung cancer metastasis in the lymph node, the different origin of A549 and NCI-H1770 was then investigated for the difference observed in A549 and NCI-H1770 cells under hypoxia. A549 cells were transfected with a HA-tagged R428W mutant TP53 plasmids. Stably selected cells were then transduced with shRNA targeting the 3'-UTR of TP53. Successful knockdown of the endogenous $(>90 \%$ knockdown was achieved) and overexpression of mutant TP53 were verified via western blot analysis assays using p53 and HA antibodies (Fig. 2B). To determine whether the regulation of PPM1D protein expression in the TP53-mutant A549 cells was such as that observed in NCI-H1770 cells (Fig. 1E and F), variants of the A549-R248W $\triangle$ TP53 cells stably expressing either a control shRNA or shRNA targeting RBM38 were generated. Successful knockdown was verified by western blot analysis (Fig. 2C). Cells expressing the control or RBM38 shRNA were either maintained under normoxic condition or subjected to hypoxia. As in NCI-H1770 cells, hypoxia 
A

\begin{tabular}{|c|c|c|c|c|c|c|c|}
\hline & $\begin{array}{l}\text { Predicted consequential pairing of target region (top) } \\
\text { and miRNA (bottom) }\end{array}$ & $\begin{array}{l}\text { Site } \\
\text { type }\end{array}$ & $\begin{array}{l}\text { Context++ } \\
\text { score }\end{array}$ & $\begin{array}{l}\text { Context++ score } \\
\text { percentile }\end{array}$ & $\begin{array}{c}\text { Weighted } \\
\text { context++ score }\end{array}$ & $\begin{array}{c}\text { Conserved } \\
\text { branch length }\end{array}$ & Р \\
\hline $\begin{array}{l}\text { Position 292-298 of PPM1D 3' UTR } \\
\text { hsa-miR-129-1-3p }\end{array}$ & $\begin{array}{l}\text { 5. }^{\prime} \text {...AGUAAUUGUGACAAUAGGGCUAA.... } \\
\text { 3. III } \quad \text { UAUGAAAAACCCCAU--UCCCGAA }\end{array}$ & $\begin{array}{l}7 \text { mer- } \\
\text { A1 }\end{array}$ & -0.36 & 98 & -0.36 & 8.715 & 0.81 \\
\hline
\end{tabular}

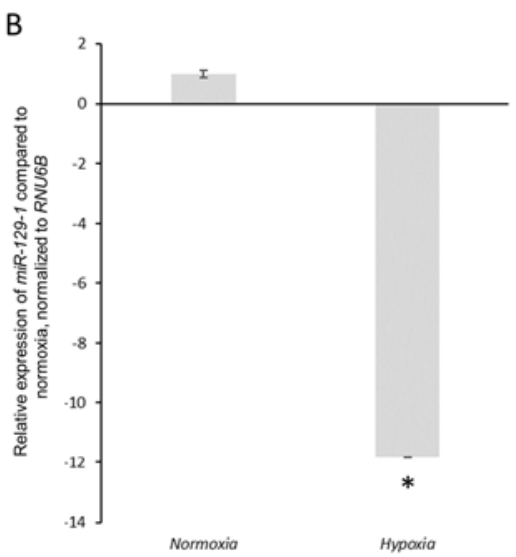

C

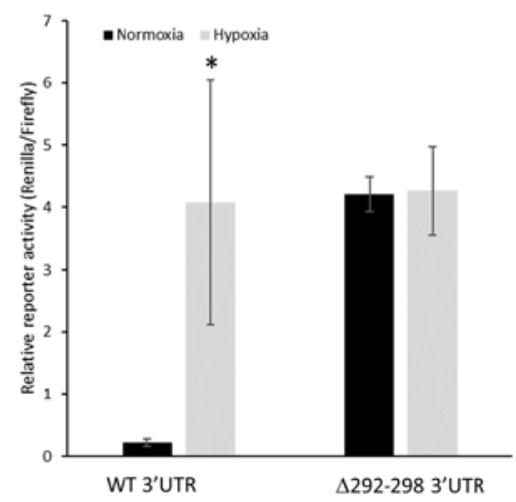

Figure 3. PPM1D is a putative target of miR-129-1-3p. (A) In situ prediction of PPMID as a potential target of miR-129-1-3p by TargetScan. (B) Relative expression of miR-129-1 in NCI-H1770 cells grown under normoxic conditions or subjected to hypoxia for $4 \mathrm{~h}$. Data were normalized to RNU6B and expressed as the mean \pm standard error of the mean. (C) Relative reporter activity of PPMID WT (WT 3'UTR) or miR-129-1-3p binding mutant 3'-UTR ( $\triangle 292-298$ 3'UTR). Data were normalized to Firefly luciferase and expressed as the mean \pm standard deviation of the ratio of Renilla and Firefly luciferase activity. "P $<0.05$. UTR, untranslated region; PPM1D, p53-induced phosphatase 1D; miR, microRNA; WT, wild type.

treatment resulted in increased PPM1D and HA-p53 protein expression (Fig. 2C, first vs. third lane). RBM38 knockdown, as in the NCI-H1770 cells, did not affect the protein expression of PPM1D or mutant p53 under hypoxia (Fig. 2C). However, when the same experiment was repeated under normoxic conditions, knockdown of $R B M 38$ resulted in significant decrease in PPM1D and increase in mutant p53 protein expression levels (Fig. 2C). Cumulatively, these results confirm that the difference observed in A549 and NCI-H1770 cells under hypoxia was not due to the different cellular origin of the NCI-H1770 and A549 cells. These results also confirm that in NSCLC cells harboring R248W mutant TP53, the induction of PPM1D protein expression is independent of RBM38 protein expression.

PPM1D is a putative target of miR-129-1-3p. TargetScan algorithm (36) was used for the prediction of potential microRNAs (miRNAs) targeting PPMID mRNA. There was only one conserved miRNA, miR-129-1-3p, predicted to target nucleotides 292-298 of the 3'-UTR of PPMID mRNA (Fig. 3A). miR-129 has previously been demonstrated to function as a tumor suppressor in lung cancer by regulating cell proliferation and metastatic progression $(37,38)$. Hence, whether PPMID is targeted by miR-129-1-3p in lung cancer cells harboring mutant TP53 and the effects on PPM1D protein expression under normoxic and hypoxic conditions were investigated.

Primarily, miR-129-1-3p expression levels in NCI-H1770 cells under normoxic and hypoxic conditions was determined. Hypoxia caused an 11.799 \pm 0.002 -fold decrease in miR-129-1-3p expression compared with normoxic conditions (Fig. 3B). To investigate whether PPMID mRNA is a direct target of miR-129-1-3p, luciferase reporter plasmids harboring either the wild-type 3'-UTR or mutant 3'-UTR (miR-129-1-3p binding site, nucleotides 292-298, deleted) were generated. These constructs were transfected in the NCI-H1770 cells and their expression was determined under normoxic and hypoxic conditions. The mutant reporter was expressed significantly higher compared with the wild-type reporter under normoxic conditions $\left(4.21 \pm 0.28\right.$ vs. $0.23 \pm 0.06$, respectively; $\left.\mathrm{P}=1.19 \times 10^{-5}\right)$ (Fig. 3C). However, following hypoxia induction, both the wild-type and mutant reporters were robustly expressed without any significant difference $(4.08 \pm 1.97$ vs. $4.25 \pm 0.71$, $\mathrm{P}=0.89$ ) (Fig. 3C).

PPM1D is targeted by miR-129-1-3p in NCI-H1770 cells under normoxia conditions. The aforementioned results indicate that the decrease in miR-129-1-3p expression under hypoxic conditions may explain the increase in protein expression of PPM1D. To test this hypothesis, control or MIR129-1-3p mimic was transiently transfected in the NCI-H1770 cells and overexpression was confirmed via RT-qPCR (Fig. 4A). The mimic transfected cells were co-transfected with the wild-type and mutant luciferase reporters and subjected to hypoxia. Reporter expression was significantly downregulated in cells transfected with miR-129-1-3p mimic. However, this was not observed with cells transfected with the control mimic (4.18 \pm 0.54 vs. $0.26 \pm 0.01$, respectively; $\mathrm{P}=3.32 \times 10^{-6}$; Fig. $4 \mathrm{~B}$ ). No significant difference was observed in the expression of the mutant reporter following transfection of the miR-129-1-3p mimic ( $4.47 \pm 0.09$ vs. $4.27 \pm 0.19, \mathrm{P}=0.41$; Fig. $4 \mathrm{~B})$. These results along with those presented in Fig. $2 \mathrm{C}$ confirm that miR-129-1-3p is targeting PPMID mRNA in lung cancer cells harboring mutant TP53 under normoxic conditions.

Co-occurrence of PPM1D/RBM38 and PPM1D/HIF1A mutations in patients with NSCLC. Based on the aforementioned 

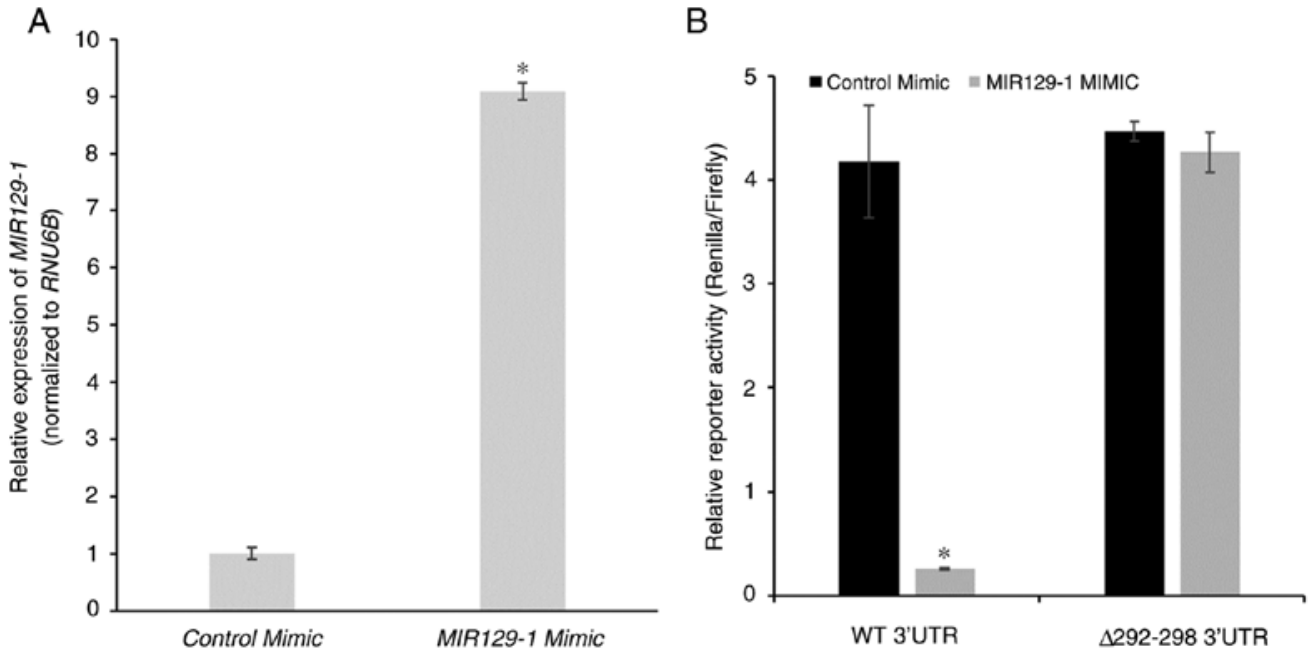

Figure 4. PPM1D is targeted by miR-129-1 in NCI-H1770 cells under normoxic conditions. (A) Relative expression of MIR-129-1in NCI-H1770 cells transiently transfected with either control or miR-129-1 mimic. Transfected cells were treated with hypoxia for $4 \mathrm{~h}$ following $48 \mathrm{~h}$ of transfection. Data were normalized to $R N U 6 B$ and expressed as the mean \pm standard error of the mean. (B) Relative reporter activity of PPM1D wild-type or miR-129-1-3p binding mutant 3'-UTR in NCI-H1770 cells transfected with control or miR-129-1 mimic. Data were normalized to Firefly luciferase and expressed as ratio of Renilla and Firefly luciferase activity. ${ }^{\text {P }}<0.05$. UTR, untranslated region; PPM1D, p53-induced phosphatase 1D; miR, microRNA; WT, wild type.

A

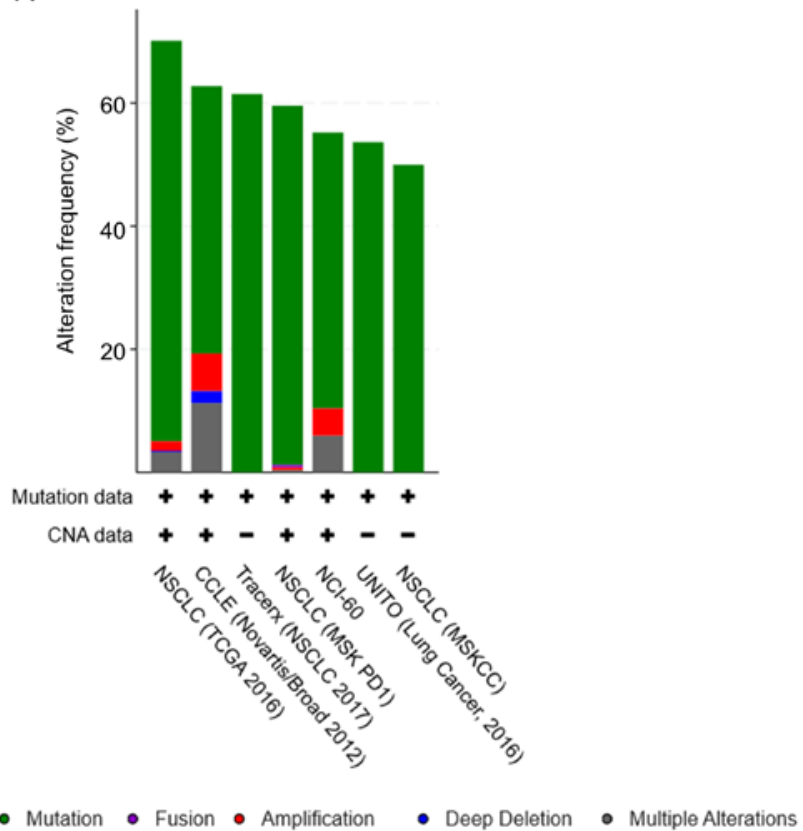

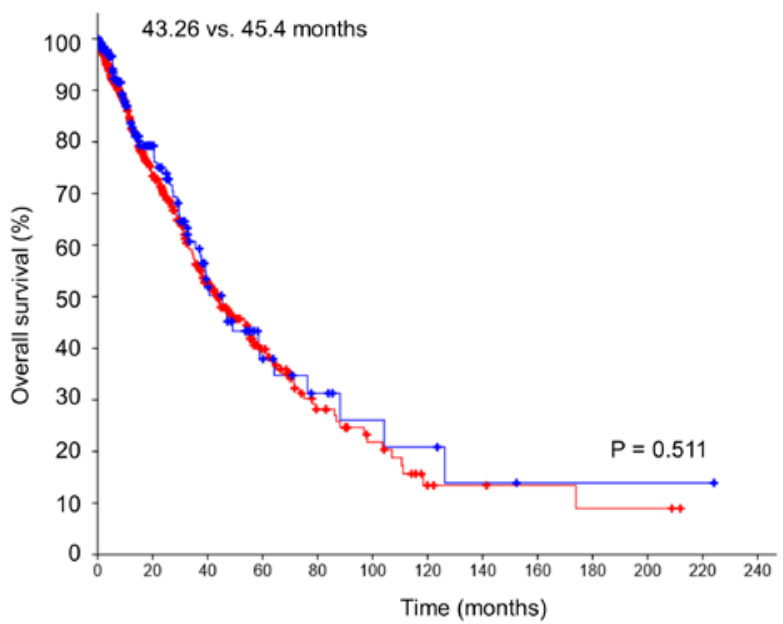

Cases with alterations in query genes $(n=695)$

Cases with no alterations in query genes $(n=259)$

Figure 5. Co-occurrence of PPM1D/RBM38 and PPM1D/HIF1A mutations in patients with NSCLC. (A) Amplification and somatic mutations of TP53, PPM1D, RBM38 and HIF1A in NSCLC cell lines and patients. Data analyzed were retrieved from TCGA and referred to 2,628 patients (2,855 samples) from 7 studies. The analysis based on the cancer studies is presented. (B) Kaplan-Meier overall survival analysis curve did not reveal any significant difference in survival times between patients with NSCLC with ( $\mathrm{n}=695$ patients) and without ( $\mathrm{n}=259$ patients) genomic amplification or somatic mutation in TP53, PPM1D, RBM38 and HIF1A. P=0.551. TCGA, The Cancer Genome Atlas; PPM1D, p53-induced phosphatase 1D; shRNA, short hairpin RNA; RBM38, RNA binding motif protein 38 ; HIF1 $\alpha$, hypoxia-inducible factor $1 \alpha$; NSCLC, non-small cell lung cancer.

observations, a TCGA dataset on NSCLC samples was analyzed to look at genomic alterations in PPM1D, RBM38, TP53, MIR129-1-3P and HIF1A in 2,628 patients $(2,855$ samples) (Fig. S1) (29-35). TP53 mutations were present in $61 \%$ of the cases, whereas RBM38, PPMID and HIF1A mutations were present in 5, 3 and $4 \%$, respectively. Most of these were point mutations, even though some genomic amplifications and deep deletions were also observed (Fig. 5A).
MIR129-1-3P mutation data were missing among the datasets selected for this study. Notably, when the tendency of co-occurrence of mutations in the aforementioned genes was examined, PPMID/HIFIA and PPMID/RBM38 exhibited significant co-occurrence $(\mathrm{P}=0.016$ and 0.029 , respectively; Table SI). RBM38 and TP53 mutations had a tendency of mutual exclusivity, even though this was not significant. Next, the difference in overall survival of patients with genomic 
alterations in PPMID, RBM38, HIF1A, and TP53 $(\mathrm{n}=695)$ and those without any alterations in these genes $(\mathrm{n}=259)$ was investigated. The median survival time was 45.4 months in patients with no alterations vs. 43.26 months in patients with alterations ( $\mathrm{P}=0.511$; Log rank t-test; Fig. 5B). These results indicate that in a small cohort of patients with NSCLC, PPMID mutations tend to co-occur with RBM38 or HIF1A; however, not both at the same time.

\section{Discussion}

In the present study, miR-129-1-3p, and not RBM38, was revealed to regulate PPM1D protein expression in mutant p53-harboring NSCLC cells under hypoxia. Under normoxic conditions, even though PPM1D expression was higher in the NCI-H1770 cells, PPM1D protein was detectable in A549 cells with wild-type p53. However, high expression of miR-129-1-3p in NCI-H1770 cells maintained under normoxic conditions was detected. miR-129-1-3p expression was downregulated under hypoxia resulting in a further induction of PPM1D protein.

Even though miR-129-1-3p levels were high in normoxic conditions in NCI-H1770 cells, there was not a complete halt in PPM1D translation. One explanation may be that RBM38 is present and is known to induce translation of PPM1D. Thus, there may be a homeostatic balance between RBM38 and miR-129-1-3p that results in decreased translation of PPMID under normoxic conditions.

Notably, despite the fact that RBM38 is required for the translation of mutant TP53 and PPMID $(1,6,8,9)$, no changes in the protein expression levels of p53 or PPM1D in NCI-H1770 cells or mutant A549 following after knockdown of $R B M 38$ were observed. One argument can be the inefficient knockdown of $R B M 38$, allowing the residual protein to bind and translate PPMID and mutant TP53 mRNAs. However, it is surprising that there was no decrease in PPM1D and p53 protein expression following the 80-90\% decrease in RBM38 protein expression. Thus, the mechanism that regulates PPM1D translation in cases where RBM38 is not present needs to be determined. Of note, RBM38 deletion is observed in tumors harboring mutant p53 $(39,40)$. A limitation of the present study was that the mutant A549 cells used were not generated by CRISPR/Cas9-mediated targeted mutation which would have been a more robust model.

Conversely, the expression level of miR-129-1-3p in normal lung epithelial cells and lung cancer cells with wild-type p53 needs to be determined. Even though HIF1 $\alpha$ is known to complex with mutant p53 alone to transcriptionally upregulate extracellular matrix component proteins (19), whether that complex is also downregulating miR-129-1-3p expression and consequently inducing PPM1D protein expression is yet to be elucidated. Finally, functional assays need to be performed to define the significance of miR-129-1-3p-mediated PPM1D regulation in NSCLC cells with mutant p53 under hypoxic conditions.

Lung cancer cells, both with wild-type or mutant p53, experience hypoxic conditions, both during initial tumorigenesis and during metastatic progression (41). PPMID is a target of p53 and a key modulator of the p53 genomic surveillance mechanism in normal cells $(1,6,7)$. RBM38 switches from a repressor of translation of $\mathrm{p} 53$ according to its phosphorylation by GSK3 $\beta$ (10) and dephosphorylation by PPM1D (8). Considering that miR-129-1-3p was demonstrated in the present study to regulate PPM1D expression under hypoxic conditions, whether and how miR-129-1-3p expression is regulated during both tumor initiation and progression, and whether it is a direct target of p53 or mutant p53-HIF1 $\alpha$ transcriptional complex, remains to be determined.

In summary, a yet undefined mechanism of miR-129-1$3 p$-mediated regulation of PPM1D protein expression in NSCLC cells with mutant p53 under hypoxic conditions was identified. Whether a similar mechanism exists in other tumor types with wild-type or mutant $\mathrm{p} 53$ remains to be determined. In addition, the mechanism by which co-occurring PPM1D/HIF1A and PPM1D/RBM38 mutations affect tumor initiation and disease progression in lung cancer patients would be of interest to investigate.

\section{Acknowledgements}

Not applicable.

\section{Funding}

The present study was supported by the Heilongjiang Province Applied Technology Research and Development Program (grant no. GZ19C01).

\section{Availability of data and materials}

All data generated or analyzed during this study are included in this published article.

\section{Authors' contributions}

HLY and QYL designed the experiments; HWX and QYL performed the experiments; HLY, HWX and QYL analyzed the experimental results. HLY wrote the manuscript. All authors read and approved the final manuscript.

\section{Ethics approval and consent to participate}

Not applicable.

\section{Patient consent for publication}

Not applicable.

\section{Competing interests}

The authors declare that they have no competing interests.

\section{References}

1. Deng W, Li J, Dorrah K, Jimenez-Tapia D, Arriaga B, Hao Q, Cao W, Gao Z, Vadgama J and Wu Y: The role of PPM1D in cancer and advances in studies of its inhibitors. Biomed Pharmacother 125: 109956, 2020.

2. Fiscella M, Zhang HL, Fan SJ, Sakaguchi K, Shen SF, Mercer WE, Vande Woude GF, O'Connor PM and Appella E: Wip1, a novel human protein phosphatase that is induced in response to ionizing radiation in a $\mathrm{p} 53$-dependent manner. Proc Natl Acad Sci USA 94: 6048-6053, 1997. 
3. Li J, Yang Y, Peng Y, Austin RJ, van Eyndhoven WG, Nguyen KC, Gabriele T, McCurrach ME, Marks JR, Hoey T, et al: Oncogenic properties of PPM1D located within a breast cancer amplification epicenter at 17q23. Nat Genet 31: 133-134, 2002.

4. Nannenga B, Lu X, Dumble M, Van Maanen M, Nguyen TA, Sutton R, Kumar TR and Donehower LA: Augmented cancer resistance and DNA damage response phenotypes in PPM1D null mice. Mol Carcinog 45: 594-604, 2006.

5. Tan DS, Lambros MB, Rayter S, Natrajan R, Vatcheva R, Gao Q, Marchiò C, Geyer FC, Savage K, Parry S, et al: PPM1D is a potential therapeutic target in ovarian clear cell carcinomas. Clin Cancer Res 15: 2269-2280, 2009.

6. Lu X, Ma O, Nguyen TA, Jones SN, Oren M and Donehower LA: The Wip1 phosphatase acts as a gatekeeper in the p53-Mdm2 autoregulatory loop. Cancer Cell 12: 342-354, 2007.

7. Lu X, Nannenga B and Donehower LA: PPM1D dephosphorylates Chk1 and p53 and abrogates cell cycle checkpoints. Genes Dev 19: 1162-1174, 2005

8. Zhang M, Xu E, Zhang J and Chen X: PPM1D phosphatase, a target of p53 and RBM38 RNA-binding protein, inhibits p53 mRNA translation via dephosphorylation of RBM38. Oncogene 34: 5900-5911, 2015.

9. Zhang J, Cho SJ, Shu L, Yan W, Guerrero T, Kent M, Skorupski K, Chen $\mathrm{H}$ and Chen X: Translational repression of p53 by RNPC1, a p53 target overexpressed in lymphomas. Genes Dev 25: $1528-1543,2011$.

10. Zhang M, Zhang J, Chen XL, Cho SJ and Chen XB: Glycogen synthase kinase 3 promotes p53 mRNA translation via phosphorylation of RNPC1. Genes Dev 27: 2246-2258, 2013.

11. Shu L, Yan W and Chen X: RNPC1, an RNA-binding protein and a target of the p53 family, is required for maintaining the stability of the basal and stress-induced p 21 transcript. Genes Dev 20: 2961-2972, 2006

12. Feldstein O, Ben-Hamo R, Bashari D, Efroni S and Ginsberg D RBM38 is a direct transcriptional target of E2F1 that limits E2F1-induced proliferation. Mol Cancer Res 10: 1169-1177, 2012.

13. Heinicke LA, Nabet B, Shen S, Jiang P, van Zalen S, Cieply B Russell JE, Xing Y and Carstens RP: The RNA binding protein RBM38 (RNPC1) regulates splicing during late erythroid differentiation. PLoS One 8: e78031, 2013.

14. Warzecha CC, Sato TK, Nabet B, Hogenesch JB and Carstens RP: ESRP1 and ESRP2 are epithelial cell-type-specific regulators of FGFR2 splicing. Mol Cell 33: 591-601, 2009.

15. Olivier M, Eeles R, Hollstein M, Khan MA, Harris CC and Hainaut P: The IARC TP53 database: New online mutation analysis and recommendations to users. Hum Mutat 19: 607-614, 2002 .

16. Freed-Pastor WA and Prives C: Mutant p53: One name, many proteins. Genes Dev 26: 1268-1286, 2012.

17. Zhang J, Xu E, Ren C, Yang HJ, Zhang Y, Sun W, Kong X, Zhang W, Chen M, Huang E and Chen X: Genetic ablation of Rbm38 promotes lymphomagenesis in the context of mutant p53 by downregulating PTEN. Cancer Res 78: 1511-1521, 2018.

18. Cho SJ, Teng IF, Zhang M, Yin T, Jung YS, Zhang J and Chen X: Hypoxia-inducible factor 1 alpha is regulated by RBM38, a RNA-binding protein and a 553 family target, via mRNA translation. Oncotarget 6: 305-316, 2015.

19. Amelio I, Mancini M,Petrova V, Cairns RA, Vikhreva P, Nicolai S, Marini A, Antonov AA, Le Quesne J, Baena Acevedo JD, et al: P53 mutants cooperate with HIF-1 in transcriptional regulation of extracellular matrix components to promote tumor progression. Proc Natl Acad Sci USA 115: E10869-E10878, 2018.

20. Zhang WC: MicroRNAs tune oxidative stress in cancer therapeutic tolerance and resistance. Int J Mol Sci 20: E6094, 2019.

21. Yu X, Song H, Xia T, Han S, Xiao B, Luo L, Xi Y and Guo J: Growth inhibitory effects of three miR-129 family members on gastric cancer. Gene 532: 87-93, 2013.

22. Bandres E, Agirre X, Bitarte N, Ramirez N, Zarate R, Roman-Gomez J, Prosper F and Garcia-Foncillas J: Epigenetic regulation of microRNA expression in colorectal cancer. Int J Cancer 125: 2737-2743, 2009.

23. Chen X, Hu H, Guan X, Xiong G, Wang Y, Wang K, Li J, Xu X, Yang K and Bai Y: CpG island methylation status of miRNAs in esophageal squamous cell carcinoma. Int J Cancer 130: $1607-1613,2012$.

24. Dyrskjot L, Ostenfeld MS, Bramsen JB, Silahtaroglu AN, Lamy P, Ramanathan R, Fristrup N, Jensen JL, Andersen CL, Zieger K, et al: Genomic profiling of microRNAs in bladder cancer: MiR-129 is associated with poor outcome and promotes cell death in vitro. Cancer Res 69: 4851-4860, 2009.
25. Schmaltz C, Hardenbergh PH, Wells A and Fisher DE: Regulation of proliferation-survival decisions during tumor cell hypoxia. Mol Cell Biol 18: 2845-2854, 1998

26. Livak KJ and Schmittgen TD: Analysis of relative gene expression data using real-time quantitative PCR and the 2(-Delta Delta C(T)) method. Methods 25: 402-408, 2001.

27. Gao J, Aksoy BA, Dogrusoz U, Dresdner G, Gross B, Sumer SO, Sun Y, Jacobsen A, Sinha R, Larsson E, et al: Integrative analysis of complex cancer genomics and clinical profiles using the cBioPortal. Science Signal 6: 11, 2013.

28. Unberath P, Knell C, Prokosch HU and Christoph J: Developing new analysis functions for a translational research platform: Extending the cBioPortal for cancer genomics. Stud Health Technol Inform 258: 46-50, 2019.

29. Barretina J, Caponigro G, Stransky N, Venkatesan K, Margolin AA, Kim S, Wilson CJ, Lehár J, Kryukov GV, Sonkin D, et al: The cancer cell line encyclopedia enables predictive modelling of anticancer drug sensitivity. Nature 483 : 603-607, 2012

30. Reinhold WC, Sunshine M, Liu H, Varma S, Kohn KW, Morris J, Doroshow $\mathbf{J}$ and Pommier Y: CellMiner: A web-based suite of genomic and pharmacologic tools to explore transcript and drug patterns in the NCI-60 cell line set. Cancer Res 72: 3499-3511, 2012.

31. Rizvi H, Sanchez-Vega F, La K, Chatila W, Jonsson P, Halpenny D, Plodkowski A, Long N, Sauter JL, Rekhtman N, et al: Molecular determinants of response to anti-programmed cell death (PD)-1 and anti-programmed death-ligand 1 (PD-L1) blockade in patients with non-small-cell lung cancer profiled with targeted next-generation sequencing. J Clin Oncol 36: 633-641, 2018.

32. Jamal-Hanjani M, Wilson GA, McGranahan N, Birkbak NJ, Watkins TBK, Veeriah S, Shafi S, Johnson DH, Mitter R, Rosenthal R, et al: Tracking the evolution of non-small-cell lung cancer. N Engl J Med 376: 2109-2121, 2017.

33. Vavalà T, Monica V, Lo Iacono M, Mele T, Busso S, Righi L, Papotti M, Scagliotti GV and Novello S: Precision medicine in age-specific non-small-cell-lung-cancer patients: Integrating biomolecular results into clinical practice-A new approach to improve personalized translational research. Lung Cancer 107: 84-90, 2017.

34. Rizvi NA, Hellmann MD, Snyder A, Kvistborg P, Makarov V, Havel JJ, Lee W, Yuan J, Wong P, Ho TS, et al: Cancer immunology. Mutational landscape determines sensitivity to PD-1 blockade in non-small cell lung cancer. Science 348: 124-128, 2015.

35. Campbell JD, Alexandrov A, Kim J, Wala J, Berger AH, Pedamallu CS, Shukla SA, Guo G, Brooks AN, Murray BA, et al: Distinct patterns of somatic genome alterations in lung adenocarcinomas and squamous cell carcinomas. Nat Genet 48: 607-616, 2016.

36. Agarwal V, Bell GW, Nam J and Bartel DP: Predicting effective microRNA target sites in mammalian mRNAs. Elife 4: e05005, 2015.

37. Li J, Wang H, Ke H and Ni S: MiR-129 regulates MMP9 to control metastasis of non-small cell lung cancer. Tumour Biol 36: 5785-5790, 2015.

38. Wu J, Qian J, Li C, Kwok L, Cheng F, Liu P, Perdomo C, Kotton D, Vaziri C, Anderlind C, et al: MiR-129 regulates cell proliferation by downregulating Cdk6 expression. Cell Cycle 9: $1809-1818,2010$

39. Wampfler J, Federzoni EA, Torbett BE, Fey MF and Tschan MP: The RNA binding proteins RBM38 and DND1 are repressed in AML and have a novel function in APL differentiation. Leuk Res 41: 96-102, 2016.

40. Leveille N, Elkon R, Davalos V, Manoharan V, Hollingworth D, Oude Vrielink J, le Sage C, Melo CA, Horlings HM, Wesseling J, et al: Selective inhibition of microRNA accessibility by RBM38 is required for p53 activity. Nat Commun 2: 513-523, 2011.

41. Kakkad S, Krishnamachary B, Jacob D, Pacheco-Torres J, Goggins E, Bharti SK, Penet MF and Bhujwalla ZM: Molecular and functional imaging insights into the role of hypoxia in cancer aggression. Cancer Metastasis Rev 38: 51-64, 2019.

This work is licensed under a Creative Commons Attribution-NonCommercial-NoDerivatives 4.0 International (CC BY-NC-ND 4.0) License. 